\section{FRI0016 \\ MUTATIONAL LANDSCAPE OF MYELODYSPLASTIC SYNDROME IN PATIENTS WITH AUTOIMMUNE RHEUMATOLOGICAL DISORDERS}

Lih En Hong ${ }^{1,2}$, Deepak Singhal ${ }^{1,3,4}$, Amilia Wee ${ }^{3,4}$, Rakchha Chhetri ${ }^{3,4}$, Monika Kutyna ${ }^{1,4}$, Mihir Wechalekar ${ }^{5,6}$, Devendra Hiwase ${ }^{1,3,4}$,

Susanna Proudman ${ }^{1,2}$. ${ }^{1}$ University of Adelaide, Discipline of Medicine, Adelaide, Australia; ${ }^{2}$ Royal Adelaide Hospital, Rheumatology Unit, Adelaide, Australia; ${ }^{3}$ Royal Adelaide Hospital, Haematology Department, Adelaide, Australia; ${ }^{4}$ South Australian Health and Medical Research Institute, Precision Medicine, Adelaide, Australia; ${ }^{5}$ Flinders Medical Centre, Rheumatology Unit, Bedford Park, Australia; ${ }^{6}$ Flinders University, Discipline of Medicine, Bedford Park, Australia

Background: Autoimmune rheumatological disorders (AIRD) have been described in myelodysplastic syndrome (MDS) and related-myeloid disorders but its association remains unclear. Immunosuppressive treatment of AIRD could cause therapy-related myeloid neoplasm (t-MN) or confound the secondary diagnosis of MDS. The prevalence of cytopenia in patients with AIRD is unknown and the mutation profile of patients with AIRD and concomitant MDS have not been well characterised.

Objectives: This study aims to evaluate the prevalence of cytopenia in AIRD and analyse the mutation profile of patients with concomitant diagnosis of AIRD and MDS by interrogating the databases of 2 large institutions - Royal Adelaide Hospital Rheumatology Department (RAH-RD) and South Australia-Myelodysplastic Syndrome (SA-MDS).

Methods: Demographic, clinical, laboratory and treatment data of 2663 patients from RAH-RD and 1157 patients from SA-MDS were analysed according to the idiopathic cytopenia of undetermined significance (ICUS) criteria. Patients with persistent cytopenia ( $>6$ months) were defined as follows: haemoglobin $<100 \mathrm{~g} / \mathrm{L}$, absolute neutrophil count $<1.8 \times 10^{9} / \mathrm{L}$ and platelet $<100 \times 10^{9} / \mathrm{L}$. Within the SA-MDS registry, 237 bone marrow samples were analysed for 43 myeloid neoplasms associated genes and 20 Fanconi (FA) DNA repair pathway genes with targeted massively parallel sequencing. An in-house filtering pipeline was used to identify somatic mutations.

Results: Within RAH-RD database, 79 patients (3\%) fulfilled the criteria for at least 1 cytopenia. 21 patients underwent bone marrow biopsy, with 9 bone marrow samples being diagnostic of MDS. Neutropenia was most common (27/79 patients, 34\%), followed by anaemia (20/79 patients, $25 \%)$ and thrombocytopenia (6/79 patients, $8 \%$ ). Within SA-MDS database, 62 patients (5\%) had concomitant AIRD. Rheumatoid arthritis is the most common AIRD diagnosis (21/62 patients, 34\%) in the SA-MDS database. Combined analysis of both databases revealed that $71 / 3820$ patients $(1.9 \%)$ had a concomitant diagnosis of both AIRD and MDS. The cytogenetic and mutational profile of patients with concomitant diagnosis of AIRD and MDS within the SA-MDS database were analysed and 56 mutations were discovered in this group. Mutation in ASXL1 was the most common $(6 / 20$ patients, $30 \%)$, followed by TET2 $(5 / 20$ patients, $25 \%)$ and lastly TP53 (4/20 patients, 20\%). There is significantly higher frequency of IDH1 mutation in the AIRD-MDS cohort compared to patients with MDS only. Three patients with TP53 mutation developed MDS following treatment for AIRD (therapy-related MDS).

Conclusion: In a large cohort of patients with AIRD, $3 \%$ of patients developed ongoing cytopenia and $0.3 \%$ were diagnosed with MDS. This finding is higher than the incidence of MDS in the general population $(0.03-0.05 \%)$. Within the SA-MDS registry, $5 \%$ of patients had concomitant diagnosis of AIRD. Our findings warrant further study and have potential implications for selection of disease-modifying drugs for patients with AIRD.

\section{REFERENCES:}

[1] Mekinian, A, et al. (2015). Systemic inflammatory and autoimmune manifestations associated with myelodysplastic syndromes and chronic myelomonocytic leukaemia: a French multicentre retrospective study. Rheumatology, 55(2), 291-300.

Disclosure of Interests: Lih En Hong: None declared, Deepak Singhal: None declared, Amilia Wee: None declared, Rakchha Chhetri: None declared, Monika Kutyna: None declared, Mihir Wechalekar Grant/research support from: Janssen \& Janssen Philadelphia USA, Devendra Hiwase Grant/research support from: Novartis, Susanna Proudman Grant/research support from: Actelion, GSK, Consultant for: Actelion, Boehringer-Ingelheim, Speakers bureau: Actelion

DOI: 10.1136/annrheumdis-2019-eular.5595

\section{FRI0017 \\ DIFFERENTIAL METHYLATION AS A PREDICTOR OF TOCILIZUMAB RESPONSE IN PATIENTS WITH RHEUMATOID ARTHRITIS}

Nisha Nair ${ }^{1}$, Darren Plant ${ }^{1,2}$, John Isaacs ${ }^{3}$, Ann Morgan ${ }^{4}$, Kimme Hyrich $^{1}$, Anne Barton ${ }^{1}$, Gerry Wilson ${ }^{5} .{ }^{1}$ The University of Manchester, Manchester, United Kingdom; ${ }^{2}$ NIHR Manchester Musculoskeletal BRC, Manchester, United Kingdom; ${ }^{3}$ NIHR Newcastle BRC, Newcastle University, Newcastle, United Kingdom;

${ }^{4}$ University of Leeds, Leeds Institute of Rheumatic and Musculoskeletal Medicine, Leeds, United Kingdom; ${ }^{5}$ University College Dublin, Dublin, Ireland

Background: Tocilizumab (TCZ) is a biological disease-modifying antirheumatic drug that blocks IL-6 signalling and is effective in ameliorating disease activity in rheumatoid arthritis (RA). Despite this, approximately $50 \%$ of patients do not respond adequately to TCZ and some patients report adverse events. Considering there is growing evidence that DNA methylation is implicated in RA susceptibility and response to some biologics (1, 2), we investigated methylation patterns as a candidate biomarker for response to TCZ in RA.

Objectives: To identify differential DNA methylation signatures in whole blood associated with TCZ response in patients with RA.

Methods: Epigenome-wide DNA methylation patterns were measured using the Infinium EPIC BeadChip (Illumina) in whole blood-derived DNA samples from patients with RA. DNA was extracted from blood samples taken pre-treatment and following 3 months on therapy, and response was determined at 6 months using the Clinical Disease Activity Index (CDAl). Patients who had good response $(n=10)$ or poor response $(n=10)$ to TCZ by 6 months were selected. Samples from secondary poor responders $(n=10)$ (patients who had an improvement of CDAl and were in remission at 3 months, followed by a worsening of CDAl at 6 months were also analysed. Differentially methylated positions (DMPs) were identified using linear regression, adjusting for gender, age, cell composition, smoking status, and steroids use.

Results: There were 20 DMPs significantly associated with response status at 6 months in the pre-treatment samples, and 21 DMPs associated with response in the 3 months samples (unadjusted P-value $<1.00 E-06$ ). Furthermore, there were $10 \mathrm{DMPs}$ significantly associated with change in methylation between pre-treatment and 3 months samples in good responders, but there were no significant findings when comparing methylation between time points in poor responders or in secondary poor responders. One DMP, cg03121467, was significantly less methylated in good responders compared to poor responders in the pre-treatment samples $(p=1.06 \mathrm{E}-06)$. Furthermore, methylation was lower in good compared with poor responders at 3 months $(p=1.03 \mathrm{E}-07)$. This DMP is close to EPB41L4A, which is thought to have a role in $\beta$-catenin signalling.

Conclusion: Whilst further analyses comparing sub-components of the CDAI with changes in methylation is ongoing, these preliminary results provide evidence that DNA methylation patterns may predict response to TCZ. Validation of these findings in other larger data sets is required.

\section{REFERENCES:}

[1] Liu,Y., Aryee,M.J., Padyukov,L., Fallin,M.D., Hesselberg,E., Runarsson,A., Reinius,L., Acevedo,N., Taub,M., Ronninger,M., et al. (2013) Epigenomewide association data implicate DNA methylation as an intermediary of genetic risk in rheumatoid arthritis. Nat. Biotechnol., 31, 142-147.

[2] Plant,D., Webster,A., Nair,N., Oliver,J., Smith,S.L., Eyre,S., Hyrich,K.L., Wilson,A.G., Morgan,A.W., Isaacs,J.D., et al. (2016) Differential Methylation as a Biomarker of Response to Etanercept in Patients With Rheumatoid Arthritis. Arthritis Rheumatol. (Hoboken, N.J.), 68, 1353-60.

Disclosure of Interests: Nisha Nair: None declared, Darren Plant: None declared, John Isaacs Grant/research support from: Pfizer, Grant/research support from: Pfizer, Consultant for: Abbvie, Pfizer, Roche, Galvani, Merck, Gilead, Eli Lilly, Amgen, Janssen, Celltrion, NAPP, Consultant for: Abbvie, Pfizer, Roche, Galvani, Merck, Gilead, Eli Lilly, Amgen, Janssen, Celltrion, NAPP, Speakers bureau: Abbvie, Pfizer, Eli Lilly, Speakers bureau: Abbvie, Pfizer, Eli Lilly, Ann Morgan: None declared, Kimme Hyrich Grant/research support from: Grants to institution: BMS, Pfizer, UCB, Anne Barton: None declared, Gerry Wilson: None declared DOI: 10.1136/annrheumdis-2019-eular.6803 